\title{
CCD imaging and aperture polarimetry of comet 2P/Encke: are there two polarimetric classes of comets?
}

\author{
K. Jockers ${ }^{1}$, N. Kiselev ${ }^{2}$, T. Bonev ${ }^{3}$, V. Rosenbush ${ }^{4}$, N. Shakhovskoy ${ }^{5}$, S. Kolesnikov ${ }^{6}$, Yu. Efimov ${ }^{5}$, \\ D. Shakhovskoy ${ }^{5}$, and K. Antonyuk ${ }^{5}$ \\ 1 Max-Planck-Institut für Sonnensystemforschung, Max-Planck-Strasse 2, 37191 Katlenburg-Lindau, Germany \\ e-mail: jockers@mps.mpg.de \\ 2 Institute of Astronomy, Karazin Kharkiv National University, Sumskaya Str. 35, 61022 Kharkiv, Ukraine \\ e-mail: kiselev@astron.kharkov.ua \\ 3 Institute of Astronomy, 1784 Sofia 72, Tsarigradsko Shose Blvd., Bulgaria \\ e-mail: tbonev@astro.bas.bg \\ ${ }^{4}$ Main Astronomical Observatory, NAS of Ukraine, Zabolotnoho Str. 27, 03680 Kyiv, Ukraine \\ e-mail: rosevera@mao.kiev.de \\ 5 Crimean Astrophysical Observatory, 98409 Nauchny, Crimea, Ukraine \\ 6 Astronomical Observatory of Odessa National University, T.G. Shevchenko Park, 65014 Odessa, Ukraine
}

Received 2 May 2005 / Accepted 9 June 2005

\begin{abstract}
We present results of imaging and aperture polarimetry of the dust of comet $2 \mathrm{P} /$ Encke at phase angles $91-105^{\circ}$, obtained during the 2003 apparition. We investigate how strongly molecular emissions transmitted by the filters used in the observations can affect the resulting polarization of cometary dust. This problem is of particular importance for so-called gasrich comets like comet $2 \mathrm{P} /$ Encke which has particularly strong molecular emission as compared to its dust continuum. Aperture polarimetry in the wide-band $U B V R$ filters was performed at the 2.6-m Shain telescope and $1.25-\mathrm{m}$ telescope of the Crimean Astrophysical Observatory on November $17-24$. From these measurements a dust polarization of $\approx 8 \%$ is derived, which puts the comet in the class of comets with low polarization. The imaging observations of comet 2P/Encke were carried out at the 2-m telescope of the Bulgarian National Astronomical Observatory on November 20-22, 2003. Narrow-band filters centered on the 0-7-0 transition of the $\tilde{A}^{2} A_{1}-\tilde{X}^{2} B_{1}$ electronic band system of $\mathrm{NH}_{2}(662 \mathrm{~nm})$ and on an adjacent red continuum at $642 \mathrm{~nm}$ were employed. The polarization of $\mathrm{NH}_{2}$ averaged over the $0-7-0$ vibronic transition amounts to $\approx 7 \%$ at phase angles close $90^{\circ}$, similar to the polarization of the two-atomic molecules $\mathrm{CN}$ and $\mathrm{C}_{2}$. The dust polarization however, when corrected for the effect of molecular emissions, is larger than $30 \%$. We conclude that the division of comets into two polarimetric classes with one class having in the visual wavelength range a maximum polarization less than $20 \%$ is caused by ignoring the contribution of molecular emission and therefore is an artifact. Whether the comet displays a strong silicate feature (i.e. its dust grains are small) or not, the dust polarization is high.
\end{abstract}

Key words. comets: individual: 2P/Encke - comets: general - polarization - methods: observational

\section{Introduction}

One of the main goals of cometary polarimetry is to determine the physical properties of cometary dust in order to learn more about its origin and formation and about the origin and formation of the comets themselves. In principle, polarimetry could be a very sensitive tool to probe the nature of cometary dust. The problem of light scattering by arbitrary dust particles, however, is still unsolved and any interpretation of polarimetric data of comets based on physical principles is uncertain as yet. Therefore, attempts have been made to find different taxonomic classes of comets by looking at their observed degree of polarization and its phase dependence. If such classes exist, one possibly could determine the ensemble properties of comets in a polarimetric class and in this way learn about the effect of these properties on polarization.
The photometric properties of comets from 0.7 to $23 \mu \mathrm{m}$ have been studied by Gehrz \& Ney (1992). These authors have grouped the investigated comets into two types, type I with a color temperature close to the blackbody and an undetectable silicate feature and type II with superheated thermal infrared continuum and high-contrast silicate feature. According to early models developed by Hanner (1980, 2003), both elevated color temperature and appearance of the silicate feature are associated with dust particle size. Therefore the two types of comets introduced by Gehrz \& Ney (1992) are mainly distinguished by their particle size (type I: large particles, and type II: small particles) but other particle properties like porosity may also be of importance. This division of comets into two classes according to their dust particle size based on infrared observations is generally accepted at present. 
Table 1. Two types of comets as defined by Gehrz \& Ney (1992) in the view of later papers.

\begin{tabular}{lll}
\hline \hline Reference & Type I & Type II \\
\hline Gehrz \& Ney (1992): & no superheat & color temperature excess \\
& no or weak silicate feature & strong silicate feature \\
& large dust grains & small dust grains \\
Chernova et al. (1993): & gas-rich & dust-rich \\
& (as apparent in visual wavelength range) \\
& low polarization? & high polarization \\
Levasseur-Regourd et al. (1996): & low polarization & high polarization \\
This paper: & high polarization & high polarization \\
\hline
\end{tabular}

In their extensive study of polarimetric properties of comets Chernova et al. (1993) noted the tendency of gas-rich comets to have lower polarization. In their paper the degree of dustiness was defined as the so-called equivalent width $W_{4845}$ introduced by Krishna Swamy (1986) and given by the ratio of light flux observed in the $5140 \AA\left(\mathrm{C}_{2}\right)$ and $4845 \AA$ filters of the International Halley Watch (IHW). This ratio is based on quantities derived from observations in the visual wavelength range where large cometary grains scatter inefficiently. Therefore it is not impossible that the comets called gas-rich by Chernova et al. (1993) in fact have the same or an even larger dust/gas mass production ratio than comets these authors called dust-rich, i.e. the denomination "gas-rich" may be misleading. Nevertheless we will use it in this paper. Only sometimes will we use the longer, but more accurate expression "so-called gasrich comets". Chernova et al. (1993) attributed the lower polarization observed in gas-rich comets mainly to the influence of molecular emission of low polarization transmitted by the continuum filters of IHW but did not exclude the possibility that part of the difference may be real. Chernova et al. (1993) did not quote Gehrz \& Ney (1992), but noted the association of their gas-rich comets with comets of low infrared color temperature and the absence of a silicate peak.

Later Levasseur-Regourd et al. (1996) grouped comets into two classes according to the maximum of their polarization phase curve. Like Chernova et al. (1993) they noted that comets of the class of high polarization display a strong silicate feature while in the group of low polarization comets the silicate feature is small or absent. Very recently a similar grouping into two polarimetric classes has been suggested from polarimetry of seven comets in the near-infrared wavelength range at $2.2 \mu \mathrm{m}$ (Kelley et al. 2004).

Summing up (see Table 1) we may associate type I comets as defined by Gehrz \& Ney (1992) (comets having large dust grains) with the gas-rich comets as defined by Chernova et al. (1993) and with the low polarization class of LevasseurRegourd et al. (1996). But the question posed already by Chernova et al. remains: is the low polarization observed in so-called gas-rich comets real or is it caused by low-polarized molecular emission transmitted by the filters used to measure the dust polarization?

In this paper we present observations of comet $2 \mathrm{P} /$ Encke obtained during its last apparition in November 2003. Comet 2P/Encke has a weak silicate feature (Gehrz et al. 1989), large dust particles (Reach et al. 2000), and is gas-rich according to the definition of Chernova et al. (1993). This would put the comet in the low polarization class. Nevertheless, as we will see, the polarization of its dust at phase angles close to $90^{\circ}$, after correction for the presence of molecular emission, exceeds $30 \%$. Apart from comet 2P/Encke there are other gas-rich comets (see Table 4 below) with high dust polarization. Therefore we will argue in our paper that the division of comets in two groups with one group having a maximum polarization in the visual wavelength range significantly less than $20 \%$ may no longer be tenable in the form proposed by Levasseur-Regourd et al. (1996) because of gas polarization contamination.

\section{Observations, instruments and data reduction}

\subsection{Overview}

Imaging polarimetry of comet $2 \mathrm{P} /$ Encke was carried out at the 2-m Ritchey-Crétien-Coudé (RCC) telescope of the Bulgarian National Astronomical Observatory (Rozhen) on November 20-22, 2003 (observers K. Jockers, T. Bonev, and G. Borisov). Aperture polarimetry of the comet was obtained with the 2.6-m Shain telescope (observers N. Kiselev, N. Shakhovskoy, and S. Kolesnikov) and 1.25-m telescope (observers Yu. Efimov, V. Rosenbush, and K. Antonyuk) of the Crimean Astrophysical Observatory on November 17-24, 2003. Weather conditions were good at both observatories. Nevertheless, the observing conditions were difficult as the comet moved rapidly at low galactic latitude in front of many field stars. In spite of the rather high total brightness $(\approx 7 \mathrm{mag})$ of comet $2 \mathrm{P} /$ Encke, its continuum was extremely weak. At the 2-m and 1.25-m telescopes a moving guide probe was used to track the comet. Table 2 gives the heliocentric and geocentric distance, phase angle (Sun-Comet-Earth angle) and the position angle of the antisolar direction (scattering plane) and filters and pixel scale or diaphragm diameter at the time of the observations.

Standard stars with small (Serkowsky 1974; Heiles 2000) and with large polarization (Hsu \& Breger 1982) were observed to determine the parameters of instrumental polarization and the zero-point of the position angle of the polarization plane for each telescope. 
Table 2. Parameters of the observations.

\begin{tabular}{|c|c|c|c|c|c|c|c|}
\hline $\begin{array}{l}\text { Date } \\
2003 \\
\text { (UT) }\end{array}$ & $\begin{array}{c}\text { Filter } \\
\lambda_{0} / F W H M \\
(\mathrm{~nm})\end{array}$ & $(\mathrm{AU})$ & $(\mathrm{AU})$ & Phase angle & $\begin{array}{c}\text { Scattering } \\
\text { plane } \\
\left({ }^{\circ}\right)\end{array}$ & $\begin{array}{c}\text { Pixel or } \\
\text { diaphragm } \\
(\operatorname{arcsec})\end{array}$ & $\begin{array}{c}\text { Scale } \\
\text { at comet } \\
\left(\mathrm{km} \operatorname{arcsec}^{-1}\right)\end{array}$ \\
\hline \multicolumn{8}{|c|}{ CCD polarimetry at 2-m telescope of the Rozhen Observatory } \\
\hline Nov. 20.746 & $642 / 2.6$ & 0.946 & 0.265 & 91.1 & 58.8 & 0.88 & 192 \\
\hline Nov. 21.750 & $642 / 2.6$ & 0.930 & 0.268 & 94.6 & 57.2 & 0.88 & 194 \\
\hline Nov. 22.738 & $642 / 2.6$ & 0.913 & 0.271 & 98.0 & 55.7 & 0.88 & 197 \\
\hline Nov. 20.751 & $662 / 5.9$ & 0.946 & 0.265 & 91.2 & 58.8 & 0.88 & 192 \\
\hline Nov. 21.767 & $662 / 5.9$ & 0.929 & 0.268 & 94.7 & 57.2 & 0.88 & 194 \\
\hline Nov. 20.700 & $694 / 79$ & 0.947 & 0.265 & 91.0 & 59.0 & 0.88 & 192 \\
\hline Nov. 21.702 & $694 / 79$ & 0.930 & 0.267 & 94.4 & 57.4 & 0.88 & 194 \\
\hline \multicolumn{8}{|c|}{ Aperture polarimetry at 2.6-m telescope of the Crimean Astrophysical Observatory } \\
\hline Nov. 21.718 & $R$ & 0.930 & 0.268 & 94.5 & 57.3 & 15 & 194 \\
\hline Nov. 23.792 & $R$ & 0.895 & 0.275 & 101.6 & 54.3 & 15 & 199 \\
\hline Nov. 24.802 & $R$ & 0.877 & 0.279 & 105.0 & 53.0 & 15 & 203 \\
\hline \multicolumn{8}{|c|}{ Aperture polarimetry at $1.25-\mathrm{m}$ telescope of the Crimean Astrophysical Observatory } \\
\hline Nov. 17.765 & $U B V$ & 0.996 & 0.261 & 80.8 & 64.4 & 30 & 189 \\
\hline Nov. 21.719 & $U B V$ & 0.930 & 0.268 & 94.5 & 57.3 & 30 & 194 \\
\hline Nov. 22.747 & $U B V$ & 0.913 & 0.271 & 98.0 & 55.8 & 30 & 196 \\
\hline Nov. 23.728 & $U B V$ & 0.896 & 0.275 & 101.4 & 54.4 & 30 & 199 \\
\hline
\end{tabular}

\subsection{Imaging polarimetry}

\subsubsection{Instrument and observations}

At Rozhen Observatory the two-channel focal reducer (2CFR) of the Max-Planck-Institut für Sonnensystemforschung (Jockers 1997a; Jockers et al. 2000) was employed. At the $2 \mathrm{~m} \mathrm{f} / 8$ telescope it provides a pixel size in the sky of 0 ' 88 . The imaging polarimeter of 2CFR has a useful field of $99 \times 99^{\prime \prime}$. It employs a split Wollaston prism located at the image of the telescope mirror (exit pupil) in the reducing optics of the focal reducer. One half of the Wollaston prism generates polarization images at position angles $0^{\circ}$ and $90^{\circ}$, and the other half at $+45^{\circ}$ and $-45^{\circ}$ (see Geyer et al. 1996; Jockers et al. 2000). In this way four polarization images are generated simultaneously on the CCD detector. Under the prevailing conditions of a high density of background stars the simultaneity of the four polarization subimages was a great advantage as either all or none of the subimages was affected by a star trail. In order to further minimize the disturbing effect of the star trails, exposure times were kept short (5 min on Nov. 21 and $10 \mathrm{~min}$ on Nov. 20 and 22).

The narrow-band filters $662\left(\mathrm{NH}_{2}, \lambda_{0}=662.1 \mathrm{~nm}\right.$, $F W H M=5.9 \mathrm{~nm}), 642$ (red continuum, $\lambda_{0}=641.6 \mathrm{~nm}$, $F W H M=2.6 \mathrm{~nm})$ and the wide-band filter $\mathrm{RX}\left(\lambda_{0}=694.0 \mathrm{~nm}\right.$, $F W H M=79.0 \mathrm{~nm})$ were used. Analysis of spectra of comet 23P/1989 N1 (Brorsen-Metcalf) and 109P/1992 S2 (SwiftTuttle) (Brown et al. 1996) and of the gas-rich comet D/1996 Q1 (Tabur) (Kiselev et al. 2001) indicate that a number of unidentified and $\mathrm{NH}_{2}$ lines of the $0-8-0$ transition of the $\tilde{A}^{2} A_{1}-$ $\tilde{X}^{2} B_{1}$ band system fall within the pass-band of the 642 filter. Therefore, alternating with the 642 filter, polarization images were taken with the filter 662 , which is centered on the $0-7-0$ transition of the $\tilde{A}^{2} A_{1}-\tilde{X}^{2} B_{1}$ band system of $\mathrm{NH}_{2}$.
We mention in passing that the labelling of the vibronic transitions of the $\tilde{A}^{2} A_{1}-\tilde{X}^{2} B_{1}$ band system of $\mathrm{NH}_{2}$, which are bent in the $\tilde{X}$ ground state but linear in the $\tilde{A}$ state, depends on which of the two states is used for the labelling. In this paper we follow the more traditional labelling related to the $\tilde{A}$ state. According to Jensen et al. (2003) the labelling of the bent $\tilde{X}$ state with respect to the labelling of the linear $\tilde{A}$ state is connected by the equation

$v_{2}^{\operatorname{lin}}=2 v_{2}^{\text {bent }}+|K+1|$,

i.e. in the nomenclature of the bent ground state the $0-7-0$ transition corresponds to $0-3-0 K=0$, and the $0-8-0$ transition to 0-3-0 K = 1 (see also Bunker \& Jensen 1998).

\subsubsection{Data reduction of imaging polarimetry}

The CCD images were processed using a program package written by K.J. in the IDL language. The image processing included bias substraction, flat field correction and removal of cosmic ray events. Point sources are selected interactively in the polarization subimages and their polarization is determined. From the standard star images the following mean parameters of instrumental polarization on Nov. 20-22 were derived: $q_{642}=-3.10 \pm 0.10 \%, u_{642}=2.78 \pm 0.06 \%, q_{662}=$ $-3.56 \pm 0.10 \%, u_{662}=2.98 \pm 0.06 \%, q_{\mathrm{RX}}=-2.44 \pm 0.11 \%$, $u_{\mathrm{RX}}=3.20 \pm 0.08 \%$. After correction for instrumental polarization, the degree of polarization of the standard star differs from the catalogue value by $0.2 \pm 0.2 \%$ and $0.3 \pm 0.2 \%$ in the filters 642 and 662 , respectively. The correction of position angle $\Delta \theta$ of the plane of polarization is equal to $1.25 \pm 0.25^{\circ}$ in all filters. To derive polarization maps of the comet the polarization subimages must be aligned. Because of the low signal/noise ratio of the individual exposures of comet Encke with 5 or 10 min duration, before determination of the polarization all 
useful exposures (without star trails in the immediate neighborhood of the comet) were aligned to a reference image (the best exposure of the night). After alignment the optical centers in the polarization subimages still sometimes differ by almost \pm 1 pixel. This acts as an additional image smearing similar to seeing effects but affects polarization only to a minor extent as the four windows are affected in a similar way. From the aligned and added subimages, intensity and polarization images $q_{\text {obs }}$ and $u_{\text {obs }}$ in the instrumental system were calculated. Areas where the total intensity had less than 30 counts were marked as areas not containing valid information and set to zero. In the last step the valid parts of $q_{\text {obs }}$ and $u_{\text {obs }}$ were corrected for instrumental polarization, and the degree of linear polarization $P_{\mathrm{obs}}=\sqrt{q_{\mathrm{obs}}^{2}+u_{\mathrm{obs}}^{2}}$ and the position angle of the polarization plane $\theta=0.5 \arctan \left(u_{\mathrm{obs}} / q_{\text {obs }}\right)$ were calculated. The errors of polarization degree $\sigma_{P}$ and angle $\sigma_{\theta}$ were determined from the photon statistics of the counts (total intensity) of comet and background contained in the sum of useful images. The following equations were used (Shakhovskoy \& Efimov 1972; Geyer et al. 1996):

$\sigma_{P}=\frac{\sqrt{I_{\mathrm{com}}+2 I_{\mathrm{backgr}}}}{I_{\mathrm{com}}} ; \quad \sigma_{\theta}=28.65 \sigma_{P} / P$.

The correction of the degree of polarization $P_{\text {true }}=\sqrt{P^{2}-\sigma_{p}^{2}}$ suggested by Wardle \& Kronberg (1974) was not applied. According to Table 3 this correction always amounts to less than $1 \%$, i.e. less than possible systematic errors.

\subsubsection{Correction for $\mathrm{NH}_{2}$ emission and its polarization}

As already mentioned, filter 642, designed to transmit only cometary continuum, transmits also part of the 0-8-0 transition of the $\tilde{A}^{2} A_{1}-\tilde{X}^{2} B_{1}$ band system of $\mathrm{NH}_{2}$ and weaker unidentified emission features. In order to be able to correct for this emission and to determine the true polarization of the cometary continuum, comet 2P/Encke was also observed with the filter 662 which transmits the $\mathrm{NH}_{2}$ 0-7-0 emission of the same $\mathrm{NH}_{2}$ band. In the following we must assume that the (faint) emissions transmitted by the 642 filter have the same spatial distribution as $\mathrm{NH}_{2}$. The reduction steps are illustrated in Figs. 1 and 2 for Nov. 21 where we have the best data. Figure 1 shows intensity and Fig. 2 polarization. In the left panels we give two-dimensional maps and in the right panels averages over the three rows closest to the nucleus. These cuts refer to the East-West direction and extend into comet Encke's fan.

In order to determine the spatial distribution and polarization of $\mathrm{NH}_{2}$ and dust from the polarization images obtained through the two filters we use the following equations:

$I_{\lambda}^{\mathrm{obs}}=I_{\lambda}^{\mathrm{d}}+I_{\lambda}^{\mathrm{g}}, \quad \lambda=642,662 \mathrm{~nm}$.

The observed intensity $I_{\lambda}^{\text {obs }}$ is the sum of the intensities of dust $I_{\lambda}^{\mathrm{d}}$ and $\mathrm{NH}_{2} I_{\lambda}^{\mathrm{g}}$, at wavelength $\lambda$.

In rows $a$ and $b$ of Figs. 1 and 2 the uncorrected "observed" data in filters 662 and 642 are displayed. The image of filter 642 (continuum) is more peaked. This indicates that the dust in comet $2 \mathrm{P} /$ Encke probably is more concentrated to the

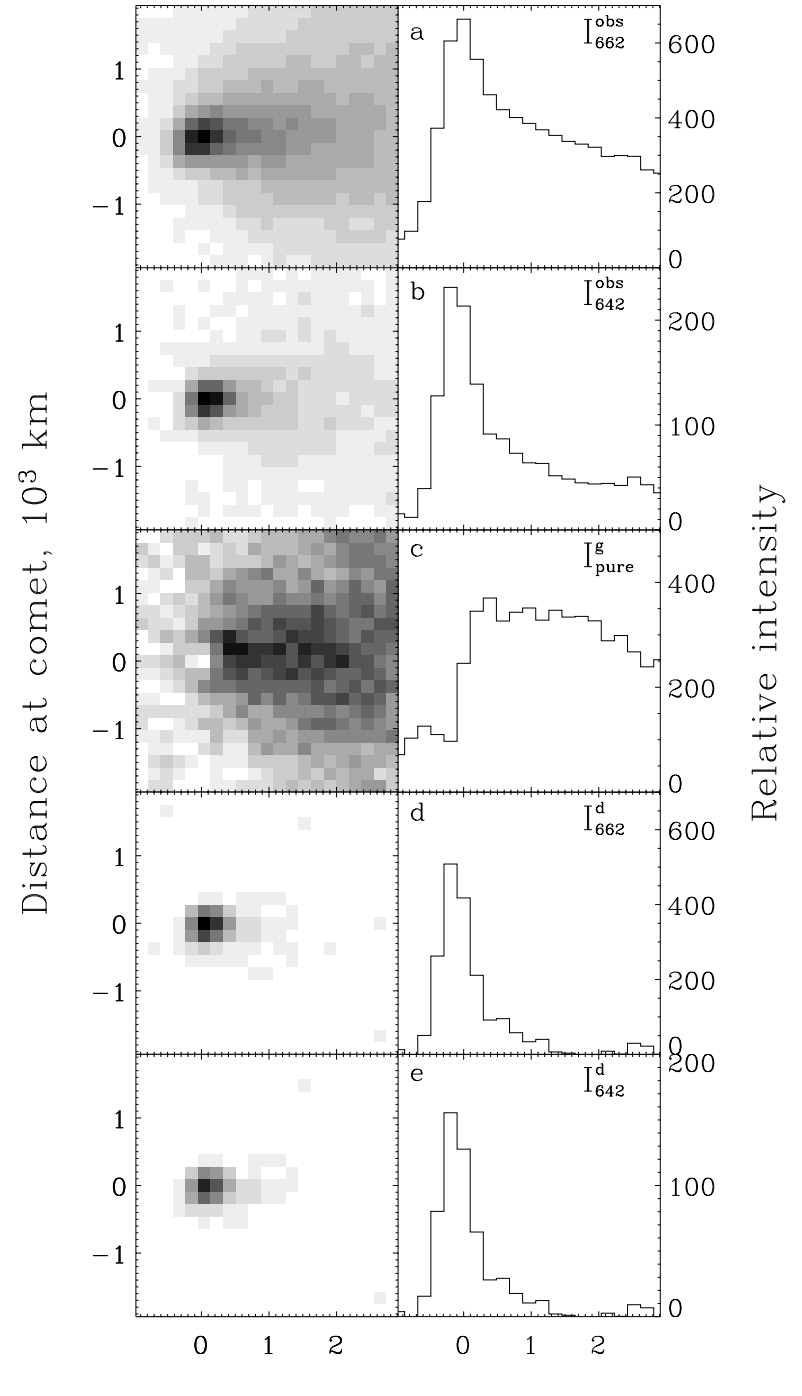

Distance at comet, $10^{3} \mathrm{~km}$

Fig. 1. Data reduction steps, intensity. Left column: images. Right column: plots of average of three central rows through comet (along fan). Rows: a) Raw image in filter 662. b) Raw image in filter 642. c) $\mathrm{NH}_{2}$. d) Dust derived from filter 662. e) Dust derived from filter 642. East is to the left, north is up.

nucleus while the $\mathrm{NH}_{2}$ gas extends into the fan. This idea is confirmed when we consider the polarization observed in filter 662 (Fig. 2, row $a$ ). Within error limits the polarization becomes constant $(7.1 \pm 0.5 \%)$ at distances into the fan larger than $\approx 1500 \mathrm{~km}$. The fact of constant polarization indicates that at larger distances into the fan only $\mathrm{NH}_{2}$ gas contributes to the observed intensity. We conclude that the polarization in the $0-7-0$ vibronic band averaged over the individual lines transmitted by the 662 filter equals $7.1 \pm 0.5 \%$.

The wavelengths of $662 \mathrm{~nm}$ and $642 \mathrm{~nm}$ are close together. Therefore we can neglect possible reddening of the cometary dust. Then the continuum contribution in both filters is proportional to their transmission integral multiplied by the solar intensity in both wavelengths. This leads to the relation

$I_{662}^{\mathrm{d}}=I_{642}^{\mathrm{d}} \cdot 3.27$ 


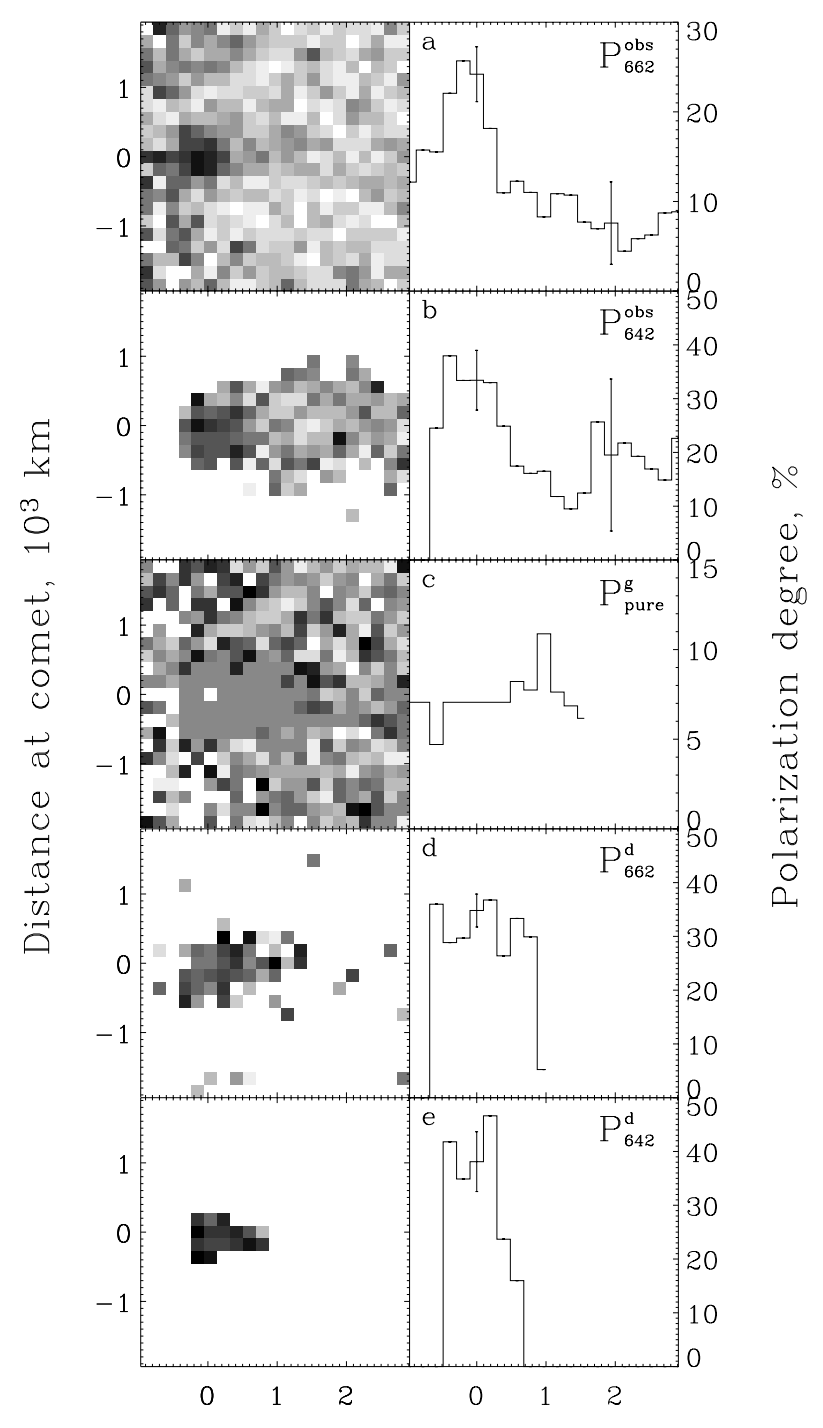

Distance at comet, $10^{3} \mathrm{~km}$

Fig. 2. Data reduction steps, polarization. Like Fig. 1, but instead of intensity the degree of polarization is shown. If the intensity (denominator) has fewer than 30 counts polarization data are considered invalid and the corresponding pixel is left blank.

Using Eq. (3) for the two wavelengths, multiplying the equation for $\lambda=642 \mathrm{~nm}$ by 3.27 and subtracting both equations, the dust contribution cancels out and we get

$I_{662}^{\mathrm{obs}}-3.27 \cdot I_{642}^{\mathrm{obs}}=I_{662}^{\mathrm{g}}-3.27 \cdot I_{642}^{\mathrm{g}}=I_{\text {pure }}^{\mathrm{g}}$.

The r.h.s. of this equation contains only the gas intensity. As long as the gas transmitted by filter 642 has the same spatial distribution as the $\mathrm{NH}_{2}$ gas transmitted by the 662 filter, the linear combination on the left side of Eq. (5) provides us with an image proportional to the pure $\mathrm{NH}_{2}$ distribution, which we denote $I_{\text {pure. }}^{\mathrm{g}}$.

Knowing from the uncorrected polarization data of filter 662 that comet Encke's pure dust coma must be less extended into the fan than the gas coma and having the pure gas image $I_{\text {pure }}^{\mathrm{g}}$ at our disposal we can now derive a pure dust image from equation

$I_{642}^{\mathrm{d}}=I_{642}^{\mathrm{obs}}-k \cdot I_{\text {pure }}^{\mathrm{g}}$.
In this equation the factor $k$ is determined from the condition that the resulting distribution is zero but not negative at large distances from the nucleus into the fan. In this way we have calculated the dust contribution $I_{642}^{\mathrm{d}}$ in the image observed in filter 642 . The gas part $I_{642}^{\mathrm{g}}$ in this filter is determined from Eq. (3) for $\lambda=642 \mathrm{~nm}$.

The dust part of the image observed in filter 662 can be calculated by multiplying $I_{642}^{\mathrm{d}}$ by the factor 3.27 (4) and the gas part again from Eq. (3), this time for $\lambda=662 \mathrm{~nm}$.

Now we can correct the observed polarization and find the true dust polarization from the equations

$$
P_{\lambda}^{\mathrm{obs}} I_{\lambda}^{\mathrm{obs}}=P_{\lambda}^{\mathrm{d}} I_{\lambda}^{\mathrm{d}}+P_{\lambda}^{\mathrm{g}} I_{\lambda}^{\mathrm{g}}, \quad \lambda=642,662 \mathrm{~nm}
$$

$P_{\lambda}^{\mathrm{d}}$ is the corrected polarization in both wavelengths. In rows $\mathrm{d}$ and e of Fig. 1 the intensity distribution of dust is shown in the filters 662 and 642, respectively. In the same rows of Fig. 2 the corresponding polarization is displayed.

For filter 662 we have determined the gas polarization and the solution of Eq. (7) is straightforward. For filter 642 (see Fig. 2, row b) the gas polarization (i.e. polarization at large distances into the fan) seems to be higher but the error bar is very large. Therefore we have assumed that the gas polarization in filter 642 is the same as in filter 662. But this assumption in principle cannot be justified as in this wavelength range the polarization of the individual $\mathrm{NH}_{2}$ lines should vary strongly from line to line. Like in OH (Le Borgne \& Crovisier 1987), also in $\mathrm{NH}_{2}$ only the rotational lines with low quantum numbers are excited. Moreover $Q$ transitions are possible which may be differently polarized (Truhins et al. 1997). In addition in the 642 filter there are unidentified lines where we have no idea of the polarization. Therefore we conclude that the degree of dust polarization derived from filter 662 (Fig. 2, row d) is more reliable and the value in row e is likely to be too high. On the other hand, the spatial distribution of polarization is better derived from filter 642 (row e) because the gas contribution in this filter is reduced.

At last, as a consistency check, using the corrected dust polarization map $P_{662}^{\mathrm{d}}$ we can derive a map of the polarization of $\mathrm{NH}_{2} P_{662}^{\mathrm{g}}$. This map is shown in the central row of Fig. 2. Of course, within error limits this map shows $P_{662}^{\mathrm{g}}=7.1 \%$ in accordance with the polarization map $P_{662}^{\text {obs }}$ at large distances from the nucleus in the fan.

\subsection{Aperture polarimetry}

The polarimetric observations at the 2.6-m Shain telescope were carried out with the one channel polarimeter (Shakhovskoy et al. 2001) operating in linear polarization measurement mode. For this, the fast rotating $(\approx 33 \mathrm{~Hz})$ halfwave plate (retarder) was placed in front of the fixed polaroid. Measurements of intensities were obtained for the $8 \times 2$ position angles of the retarder in the order $0^{\circ}, 22.5^{\circ}, 45^{\circ}, \ldots, 157.5^{\circ}$ with a total integration time of $4 \mathrm{~s}$ (133 rotations). One full exposure of the comet consisted of 64 integrations of $4 \mathrm{~s}$ each, i.e. $256 \mathrm{~s}$. Between exposures the sky background was measured. We used the $R$ wide-band filter and a circular diaphragm of $15^{\prime \prime}$ diameter. In absence of an offset guide probe the central condensation of the comet was centered by eye in the diaphragm. 
Table 3. The mean observed and corrected degree of polarization in comet $2 \mathrm{P} /$ Encke.

\begin{tabular}{|c|c|c|c|c|c|c|c|}
\hline \multirow{3}{*}{$\begin{array}{l}\text { Date } 2003 \\
\text { (UT) }\end{array}$} & \multirow{3}{*}{$\begin{array}{c}\text { Phase angle } \\
\left({ }^{\circ}\right)\end{array}$} & \multirow{3}{*}{$\begin{array}{l}\text { Area } \\
\left(\mathrm{km}^{2}\right)\end{array}$} & \multicolumn{5}{|c|}{ Polarization degree $(\%)$} \\
\hline & & & \multicolumn{2}{|c|}{642} & \multicolumn{2}{|c|}{662} & \multirow{2}{*}{$\begin{array}{c}\mathrm{RX} \\
\text { observed }\end{array}$} \\
\hline & & & observed & dust only & observed & dust only & \\
\hline Nov. 20.7 & 91.12 & $576 \times 576$ & $33.3 \pm 2.6$ & $41.6 \pm 6.2$ & $18.1 \pm 0.6$ & $42.9 \pm 7.8$ & \\
\hline Nov. 21.7 & 94.63 & $582 \times 582$ & $34.9 \pm 1.7$ & $39.9 \pm 2.9$ & $22.7 \pm 1.3$ & $31.1 \pm 1.2$ & $25.1 \pm 1.0$ \\
\hline Nov. 22.7 & 98.04 & $591 \times 591$ & $35.8 \pm 3.6$ & & & & \\
\hline
\end{tabular}

During one exposure the comet moved about 4 " with regard to the centre of diaphragm. Thus, the real diaphragm projected at the comet was equal of about $15 \times 23^{\prime \prime}$ with the long dimension in the direction of the proper motion of the comet in the sky.

The five-color photopolarimeter, developed at the Observatory of the University of Helsinki (Piirola 1973, 1988), is standard equipment for polarization measurement at the $1.25-\mathrm{m}$ telescope. The instrument was operated in linear polarization mode. In this instrument a rotating half-wave plate (retarder) with $22.5^{\circ}$ step in front of a fixed calcite plate (polarizer) allows to measure the two orthogonally polarized intensities simultaneously. An important advantage of this instrument for the observation of faint point sources is that the sky polarization, caused e.g. by moonlight, is eliminated and only the intensity of the sky background must be taken into account. Nevertheless, the polarimeter is not suited for measurements of extended objects like comets because of superposition of the ordinary and extraordinary images. To avoid this we had to put an additional polaroid behind the calcite plate so as to extinguish one of two orthogonally polarized intensities. A single measurement consists of eight integrations in the different orientations of the retarder. Typically the integration time was $10 \mathrm{~s}$. The full set of cometary measurements usually consisted of several cycles, each of 7-10 single measurements. The sky background radiation was measured before and after each cycle of cometary measurements. The photometric system is realized with four dichroic filters, which separate the light into five spectral regions, i.e. the instrument provides simultaneous measurements of polarization in the UBVRI bands (360, 440, 530, 690, and $830 \mathrm{~nm}$ respectively). The passbands are close to the standard $U B V$ (Johnson) and $R I$ (Cousins) systems. Unfortunately, because of low signal/noise ratio no reliable polarimetric data could be obtained in the $R$ and $I$ bands.

Before starting an exposure with either one of the aperture polarimeters the sky was visually checked for the appearance of brighter stars in the diaphragm and, if necessary, the telescope was moved away from the comet to measure the background polarization. Scans with elevated total intensity (indicating the presence of a field star in the diaphragm) were removed during data reduction.

For the five-color polarimeter the maximum of instrumental polarization was $2.08 \pm 0.08 \%, 1.26 \pm 0.07 \%$ and $1.31 \pm 0.05 \%$ in the $U B V$ filters, respectively, while the instrumental polarization for aperture observations at the 2.6-m telescope did not exeed $0.02 \pm 0.05 \%$. The main contribution to the errors in polarization degree of $2 \mathrm{P} /$ Encke comes from the limited signal to noise ratio for the continuum of the comet. The degree of polarization $P$ and the position angle $\theta$ were calculated as in the case of the imaging polarimetry. The errors of the Stokes parameters $q$ and $u$ were determined from both the statistics of recorded photons (as with imaging polarimetry) and from the scatter of individual measurements (Shakhovskoy \& Efimov 1972). The larger of these two errors was adopted as the accuracy measure.

\section{Results of polarization measurements}

\subsection{Imaging polarimetry}

Table 3 provides the degree of polarization derived from the imaging polarization measurements. The values are averaged over $3 \times 3$ pixel centered on the nucleus (i.e. the pixel with maximum count rate in the intensity image). We provide the observed values in the interference filters 642,662 and in the wide-band filter RX and the values corrected for the presence of molecular emission in the filter passband. These values are labeled "dust only". As explained above, a correction for molecular emission is only possible if observations in both filters 642 and 662 are available. The degree of polarization measured in the red wide-band filter cannot be corrected.

For all observations the plane of polarization of the comet was perpendicular to the plane of scattering within observation errors. Therefore, in Table 3 and the following tables we do not provide the position angle of the plane of polarization. One can see that the polarization of the dust of gas-rich comet $2 \mathrm{P} /$ Encke at phase angles $\approx 90^{\circ}$ exceeds $30 \%$ i.e. the dust of the comet has a polarization higher than the average polarization of so-called dust-rich comets (see Fig. 3 below).

\subsection{Aperture results}

The results of photoelectric measurements of polarization through wide-band filters and large diaphragms are given in Table 4 . The low observed integrated polarization of $\approx 8 \%$ in the wide-band $U, B, V$, and $R$ filters is caused by the emission of the radicals $\mathrm{CN}, \mathrm{C}_{3}, \mathrm{C}_{2}$, and $\mathrm{NH}_{2}$ which according to Osip et al. (1992) and Fink \& Hicks (1996) are the predominant molecular components in the spectrum of comet $2 \mathrm{P} /$ Encke. The polarization of molecules $\mathrm{CN}$ and $\mathrm{C}_{2}$ and $\mathrm{C}_{3}$ and its phase angle dependence is well studied theoretically (Le Borgne \& Crovisier 1987 , and references therein). The phase dependence of polarization is described by the expression:

$p(\alpha)=\frac{p_{90} \cdot \sin ^{2} \alpha}{1+p_{90} \cdot \cos ^{2} \alpha}$, 
Table 4. Aperture polarization results for comet $2 \mathrm{P} /$ Encke.

\begin{tabular}{lccrr}
\hline \hline $\begin{array}{l}\text { Nov. } \\
2003 \\
(\mathrm{UT})\end{array}$ & Filter & Area & $\begin{array}{c}\text { Phase } \\
\text { angle } \\
\left({ }^{\circ}\right)\end{array}$ & \multicolumn{1}{c}{$P \pm \sigma P$} \\
\hline \multicolumn{5}{c}{$2.6-\mathrm{m}$ telescope } \\
\hline 21.718 & $R$ & $2916 \times 4471$ & 94.52 & $7.7 \pm 1.8$ \\
23.792 & $R$ & $2991 \times 4587$ & 101.63 & $6.7 \pm 1.0$ \\
24.718 & $R$ & $3035 \times 4654$ & 105.01 & $9.8 \pm 1.1$ \\
\hline \multicolumn{5}{c}{1.25 -m telescope } \\
\hline 17.765 & $U$ & $\pi \times 5679^{2}$ & 80.75 & $8.06 \pm 0.12$ \\
& $B$ & & $7.66 \pm 0.11$ \\
& $V$ & & $10.42 \pm 0.12$ \\
21.719 & $U$ & $\pi \times 5831^{2}$ & 94.51 & $7.23 \pm 0.14$ \\
& $B$ & & $16.40 \pm 0.58$ \\
& & & $5.00 \pm 0.11$ \\
22.747 & $U$ & $\pi \times 5896^{2}$ & 98.04 & $7.48 \pm 0.09$ \\
& $B$ & & $7.85 \pm 0.08$ \\
& $V$ & & $5.53 \pm 0.09$ \\
23.728 & $U$ & $\pi \times 5983^{2}$ & 101.42 & $7.28 \pm 0.08$ \\
& $B$ & & & $7.43 \pm 0.08$ \\
& $V$ & & $9.12 \pm 0.07$ \\
\hline
\end{tabular}

where $p_{90}$ is the maximum polarization at phase angle $\alpha=90^{\circ}$. For the bands of $\mathrm{CN}$ and $\mathrm{C}_{2} p_{90}=0.077$, while for the $\mathrm{C}_{3}$ polarization $p_{90}=0.19$ is expected. For the diatomic molecules $\mathrm{CN}$ and $\mathrm{C}_{2}$ the observations (Le Borgne et al. 1986; Le Borgne $\&$ Crovisier 1987; Kiselev 2003) are in good agreement with the theoretical calculations. For $\mathrm{C}_{3}$, however, the polarization degree observed by Le Borgne et al. (1986) was lower than the theoretical value. $\mathrm{C}_{3}$ emission at $\approx 405 \mathrm{~nm}$ mostly affects the $B$ band. On Nov. 21.7 (but only then) a polarization of $16.4 \%$ was observed. On the other days the observed polarization was closer to the values of $\mathrm{CN}$ and $\mathrm{C}_{2}$, also present in the $B$ band. As mentioned in Sect. 2.2.3, for filter 662 a polarization of $\mathrm{NH}_{2}$ of $\approx 7 \%$ was deduced from our imaging polarimetry. But we do not know what value we might expect for the polarization of the gas emissions integrated over the whole $R$ band.

\section{Discussion}

Figure 3 shows the polarization of comet $2 \mathrm{P} /$ Encke derived from imaging polarimetry for several square apertures and from photoelectric observations. Data from the previous paper (Kiselev et al. 2004) for an area of $2000 \mathrm{~km}^{2}$ are also included. The full line presents the phase angle dependence of polarization for resonance fluorescence according to Eq. (8). The dashed line is the "standard" phase curve of polarization of dusty comets (Kiselev 2003; see also Jockers 1997b) in the red continuum filter $(684.0 / 9.0 \mathrm{~nm})$, calculated from the fit

$P(\alpha)=32.049 \sin ^{0.825}(\alpha) \cos ^{0.425}(\alpha / 2) \sin \left(\alpha-21.8^{\circ}\right)$.

In the wide-band filters the degree of polarization observed in comet 2P/Encke, when averaged over a large coma area, is close to the value for resonance fluorescence of $\mathrm{CN}$ and $\mathrm{C}_{2}$. The values agree well with polarization measurements of other gas-rich comets like C/1975 N1 (Kobayashi-Berger-Milon),

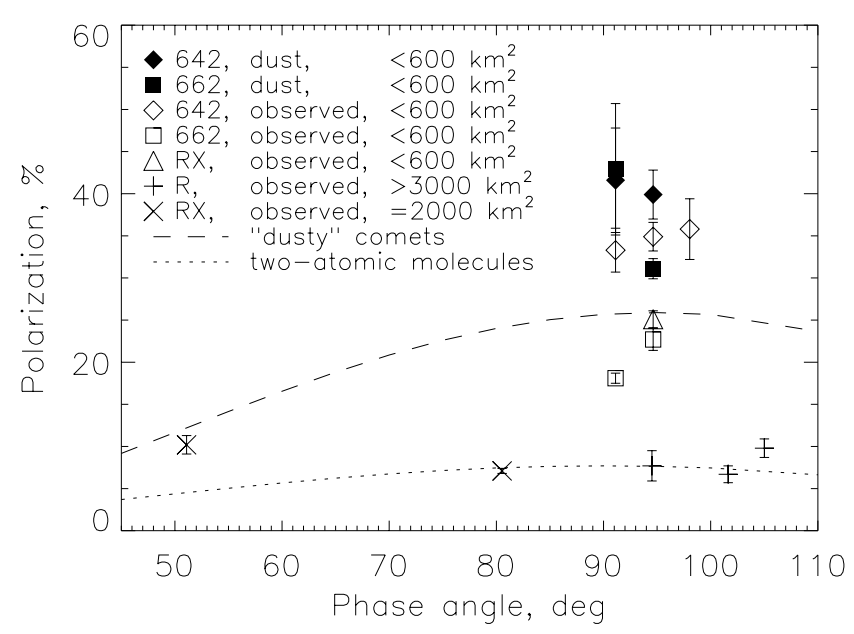

Fig. 3. Degree of polarization of comet 2P/Encke in the red spectral range obtained in different diaphragms as compared to two-atomic molecules (Eq. (8), dotted line) and typical dusty comets (red spectral range, Eq. (9), dashed line). Open symbols, $\times$, and + : measurements. Filled symbols: values corrected for the influence of gas emissions in the filter passband. All symbols refer to imaging polarimetry of this paper, with two exceptions: +: aperture measurements at the $2.6-\mathrm{m}$ telescope, this paper. $\times$ : imaging polarimetry (Kiselev et al. 2004).

23P/Brorsen-Metcalf (Chernova et al. 1993), and C/1982 M1 (Austin) (Rosenbush et al. 1997) observed in wide-band filters and/or through large diaphragms. In that sense we can confirm that comet $2 \mathrm{P} /$ Encke belongs to the group of comets with low polarization at phase angles close to $90^{\circ}$. However, as one can see from Table 3 and Fig. 3, after correction for the molecular emission transmitted by the "continuum" filters, the polarization in comet $2 \mathrm{P} /$ Encke is even higher than that for so-called dust-rich comets. This possibility was already discussed by Chernova et al. (1993), in their paper about polarimetric measurements of 13 comets observed with an aperture polarimeter on Mount Sanglok, Tadjikistan. For comet 2P/Encke Kiselev et al. (2004), using measurements through a wide-band filter and correcting them for molecular emission by assuming a circular gas coma, predicted high values of dust polarization. This prediction is confirmed by observations of Jewitt (2004) and by the measurements of the present paper. Comet 2P/Encke, therefore, does not belong to the group of gas-rich comets of low polarization postulated by Levasseur-Regourd et al. (1996), as the low polarization observed in comet Encke is produced by the contribution of molecular emissions transmitted by the "continuum" filter and does not represent the true polarization of its cometary dust.

It is very likely that the low polarization measured in other gas-rich comets also is an artifact caused by molecular emissions transmitted by the filters used for the cometary observations. The improved sensitivity of astronomical spectrographs caused by the advent of the sensitive CCD-detectors has considerably increased the number of known emission lines in cometary spectra (Arpigny 1995; Brown et al. 1996). Many of them are still unidentified. As we know now, it is virtually impossible to find spectral continuum windows entirely free of cometary molecular emissions. As was recently shown by Kiselev et al. (2001), even the special narrowband continuum 
filters transmit a significant number of faint molecular emission lines. When the intrinsic continuum caused by the light scattering of cometary dust is weak, the contribution of these lines can significantly reduce the observed polarization even if the intrinsic polarization of the light scattered by the dust particles is high. Attempts of some investigators (Eaton et al. 1992; Levasseur-Regourd et al. 1996) to carry out polarimetric measurements with broad-band filters in the red spectral region did not improve the situation because faint molecular lines are present also in this part of the cometary spectrum. Here the most important molecules are $\mathrm{NH}_{2}$ and $\mathrm{H}_{2} \mathrm{O}^{+}$.

The second difficulty of measurements of the intrinsic dust polarization is related to the low spatial resolution of most aperture observations of comets. The small dust grains contributing most to the visible light scattered by cometary dust are released from the nucleus. Even if they are altered by fractionation or loss of volatiles their distribution peaks sharply at the cometary nucleus. Cometary neutrals observable in the visual wavelength range, however, are daughter molecules. Close to the nucleus they have a shallower distribution than cometary dust grains. Therefore it is expected that in gas-rich comets, because the gas/dust ratio increases with distance from the nucleus, if the filters transmit molecular emissions, the observed polarization will decrease with distance from the nucleus and, in case of aperture measurements, with increasing aperture of the polarimeter. A good example is provided by the observations of gas-rich Comet D/1996 Q1 (Tabur) (Jockers 1997b; Kiselev et al. 2001). Instead of a gas coma surrounding the nucleus from all sides comet $2 \mathrm{P} /$ Encke has a gas jet pointing toward West. Nevertheless our observations clearly demonstrate that the observed polarization is reduced when observed with a larger diaphragm.

The polarization data available in the literature for gasrich comets in the red spectral region are collected in Table 5 together with the corresponding infrared data. In this table from left to right the columns indicate the name of the comet, the phase angle $\alpha_{\text {obs }}$ of the observation and the corresponding degree of polarization $P_{\mathrm{obs}}$, the diameter of diaphragm or size of box projected on the comet, and the typical degree $P_{\text {dust }}$ of polarization observed in dust-rich comets. This column was calculated from the trigonometric fit for dust-rich comets (Eq. (9)). Comets with large difference between observed polarization and polarization calculated from Eq. (9) are attributed to the class of low polarization. At first sight it seems indeed that gas-rich comets belong to the class of low-polarization comets as postulated by Levasseur-Regourd et al. (1996). But there are exceptions besides comet 2P/Encke. Comet C/1983 H1 (Iras-Araki-Alcock) approached the Earth very closely. Because of this even a polarimeter with a mediumsize diaphragm would pick up only the innermost part of the coma. A high polarization was observed despite the fact that this comet showed almost no dust continuum or silicate feature. Other gas-rich comets, C/1982 M1 (Austin), C/1989 Q1 (Okasaki-Levy-Rudenko) and D/1996 Q1 (Tabur), when observed through small diaphragms, showed high polarization. We are forced to dismiss the temptingly simple idea that the dust of gas-rich comets has low intrinsic polarization, and must face the fact that the large grains, constituting the majority of dust present in gas-rich comets, have a polarization as high as the grains in dust-rich comets or possibly even higher.

If we accept the idea discussed earlier that so-called gasrich comets have predominantly large dust grains, how can it be possible that large cometary dust grains have high polarization? There is further observational evidence that large dust grains can be highly polarized. When comet D/1999 S4 (LINEAR) disintegrated it left behind a highly reddened cloud of dust (Bonev et al. 2002), which could be observed for several days. When this cloud was visible, the phase angle decreased from a maximum of $\approx 120^{\circ}$ to $\approx 100^{\circ}$. There cannot be any doubt that the cloud consisted of large particles, as small particles are quickly removed by solar radiation pressure. From aperture polarimetry (Kiselev et al. 2002) a polarization of $30 \%$ was observed at a phase angle of $104^{\circ}$. Imaging polarimetry (Hadamcik \& Levasseur-Regourd 2003) indicates even higher polarization values. At a phase angle of $109^{\circ}$ (slightly earlier than the measurement by Kiselev et al.) a maximum polarization of $40 \%$ was observed on the sunward side of the remainig dust cloud.

The polarization properties of cometary dust particles are still not well understood from the theoretical side. The most successful models describe cometary dust as aggregates of constituent particles of submicron size (West 1991; Xing \& Hanner 1997; Petrova et al. 2000; Kimura 2001; Tishkovets et al. 2004; Petrova et al. 2004; Mann et al. 2004). With the exception of Xing \& Hanner (1997) all quoted papers have used spheres as constituent particles. Unfortunately, aggregate particles with sizes large compared to the wavelength, which could model realistic cometary dust grains, cannot be calculated with presently available algorithms and computers. At phase angles around $90^{\circ}$ existing aggregate models of cometary dust grains predict a polarization of $\approx 50 \%$ or higher for grains comparable to or slightly larger than the wavelength. For aggregates of larger size the polarization may decrease. The polarization is also likely to decrease for all aggregates irrespective of their size, if the constituent particles are nonspherical and have a wide size distribution. So we conclude that at present models of cometary dust grains do not contradict the idea of the existence of large cometary dust grains with a degree of polarization between $30-40 \%$. The reason for this may be that, besides the dependence on refractive index, polarization is sensitive to surface structure and compactness of the dust grains on scales comparable to the wavelength but to a much lesser extent to particle size. In this respect the observations of Kelley et al. (2004), seemingly confirming the existence of two polarimetric classes in the near infrared spectral region, are of great importance. It seems, however, premature to include them into the discussion of this paper. More work is needed on cometary spectroscopy and polarimetry of molecules and dust in the $K$ band.

\section{Conclusions}

During its apparition in November 2003 polarimetric observations of comet $2 \mathrm{P} /$ Encke were carried out at the Bulgarian National Observatory (Institute of Astronomy, Bulgarian Academy of Sciences) and the Crimean Astrophysical 
Table 5. Polarization and infrared emission data for gas-rich comets observed at large phase angles.

\begin{tabular}{|c|c|c|c|c|c|c|c|}
\hline Comet name & $\begin{array}{l}\alpha_{\mathrm{obs}}^{1} \\
\left({ }^{\circ}\right)\end{array}$ & $\begin{array}{l}\text { Diaphragm size } \\
\text { or box }\left(\mathrm{km}^{2}\right)\end{array}$ & $\begin{array}{l}P_{\text {obs }}^{2} \\
(\%)\end{array}$ & $\begin{array}{l}P_{\text {dust }}^{3} \\
(\%)\end{array}$ & $\begin{array}{l}\text { Polar. } \\
\text { class }\end{array}$ & $\begin{array}{l}\text { Overheat/silicate } \\
\text { features at } 10 \mu \mathrm{m}\end{array}$ & Ref. $^{4}$ \\
\hline $\begin{array}{l}\text { C/1975 N1 (Kobayashi- } \\
\text { Berger-Milon) }\end{array}$ & 95.4 & $\pi \times 38360^{2}$ & 9.1 & 25.9 & low & $1.08 / \mathrm{no}$ & 1,2 \\
\hline 23P/Brorsen-Metcalf & 86.0 & $\pi \times 37660^{2}$ & 15.3 & 25.2 & low & $1.05 / \mathrm{no}$ & 1,3 \\
\hline C/1982 M1 (Austin) & $\begin{array}{l}94.1 \\
98\end{array}$ & $\begin{array}{l}\pi \times 45000^{2} \\
2452 \times 5655\end{array}$ & $\begin{array}{l}9.1 \\
22.3\end{array}$ & $\begin{array}{l}25.9 \\
25.8\end{array}$ & $\begin{array}{l}\text { low } \\
\text { high }\end{array}$ & $? / ?$ & $\begin{array}{l}4,- \\
5,-\end{array}$ \\
\hline $\begin{array}{l}\text { C/1983 H1 (Iras- } \\
\text { Araki-Alcock) }\end{array}$ & 92.5 & $\pi \times 800^{2}$ & 32 & 25.8 & high & $1.07 /$ very weak & 6,7 \\
\hline 27P/Crommelin & 73.7 & $\pi \times 28760^{2}$ & 11.3 & 22.2 & low & $<1.04 /$ no & 1,8 \\
\hline C/1989 X1 (Austin) & 89.3 & $\pi \times 5400^{2}$ & 18.0 & 25.6 & low & 1.07-1.20/weak & 9,10 \\
\hline $\begin{array}{l}\text { C/1989 Q1 (Okazaki- } \\
\text { Levy-Ludenko) }\end{array}$ & 89.8 & $\pi \times 3630^{2}$ & 16.0 & 25.6 & low & $1.20 /$ no & 11,7 \\
\hline D/1996 Q1 (Tabur) & 83.3 & $\begin{array}{l}990 \times 990 \\
7422 \times 7422\end{array}$ & $\begin{array}{l}25.4 \\
15.9\end{array}$ & 24.7 & $\begin{array}{l}\text { high } \\
\text { low }\end{array}$ & $\approx 1.05 /$ weak & 12,13 \\
\hline 2P/Encke & 94.6 & $\begin{array}{l}582 \times 582 \\
\approx 3000 \times 4500\end{array}$ & $\begin{array}{l}\geq 30 \\
7.7\end{array}$ & 25.9 & $\begin{array}{l}\text { high } \\
\text { low }\end{array}$ & $1.03 /$ weak & 14,15 \\
\hline 21P/Giacobini-Zinner & 62.3 & $35300 \times 35300$ & 15.7 & 17.6 & high & weak/weak & 16,17 \\
\hline
\end{tabular}

\footnotetext{
1) Observed phase angle.

2) Observed degree of polarization.

3) Polarization of a typical dusty comet at phase angle $\alpha_{\text {obs }}$.

4) References: (1) Chernova et al. (1993); (2) Ney (1982); (3) Lynch et al. (1992); (4) Rosenbush et al. (1997);

(5) Myers \& Nordsiek (1984); (6) Kikuchi (1983); (7) Hanner et al. (1994); (8) Hanner et al. (1985);

(9) Kikuchi et al. (1990); (10) Hanner et al. (1993); (11) Rosenbush et al. (1994); (12) Kiselev et al. (2001);

(13) Harker et al. (1999); (14) This work; (15) Gehrz et al. (1989); (16) Kiselev et al. (2000);

(17) Hanner et al. (1992).
}

Observatory, Ukraine (CrAO). Imaging polarimetry was obtained in Bulgaria and aperture measurements were performed at $\mathrm{CrAO}$. The aim of the study was to verify the existence of two polarimetric classes of comets, i.e. a class of gasrich, low-polarization comets versus a class of dust-rich highpolarization comets. Comet $2 \mathrm{P} /$ Encke was an appropriate target, as it is a well studied gas-rich comet and its dust grains are known to be large. For the imaging observations we used two adjacent filters. The filter 642 (center wavelength in nanometers) transmits a so-called continuum window, and the filter 662 is centered on one of the main emissions of the $\mathrm{NH}_{2}$ radical found at $662 \mathrm{~nm}$. As was shown, the polarimetric and photometric data in the two filters allow us to unambiguously separate gas and dust in comet $2 \mathrm{P} /$ Encke. The data reduction steps are illustrated in Figs. 1 (photometry) and 2 (polarimetry). The aperture polarimetry was performed in the classical way through wide-band filters. The following results were obtained.

1. As already suggested by Festou \& Barale (2000) the famous "sunward" jet of $2 \mathrm{P} /$ Encke consists mostly of gas emission. While Festou \& Barale use spectra and one wideband image we have derived images where gas and dust are unambiguously separated. Sekanina (1988) erroneously attributed Encke's fan to dust particles.

2. The wide-band aperture polarimetric measurements done at $\mathrm{CrAO}$ result in a low polarization of comet $2 \mathrm{P} /$ Encke and seemingly confirm comet $2 \mathrm{P} /$ Encke as a member of a hypothetical class of low-polarization comets.
3. Comet $2 \mathrm{P} /$ Encke's dust in the red spectral region is almost spherically symmetric around the nucleus with a slight extension into the fan. The absence of a dust tail pointing in the antisolar direction indicates that the dust grains must be large enough not to be affected by solar radiation pressure.

4. The $\mathrm{NH}_{2}$ coma does not surround the nucleus (i.e. the optical center of the dust coma) but is somewhat detached and extends in the direction of the fan.

5. The average polarization of the $0-7-0$ transition of the $\tilde{A}^{2} A_{1}-\tilde{X}^{2} B_{1}$ band system amounts to $\approx 7 \%$, but one must expect that the polarization of individual rotational lines stronly deviates from this average.

6. In contrast to the wide-band aperture polarimetric measurements, the narrow-band imaging polarimetry, after correction for molecular emission lines transmitted by the filters, yields a degree of polarization of $\approx 40 \%$. Similar high values, but with considerably larger error bars, have been found by Jewitt (2004).

7. Apparently low values of polarization observed in other socalled gas-rich comets are caused by low polarized molecular emission transmitted by the filter employed and/or insufficient spatial resolution provided by the aperture of the polarimeter, as the molecular coma in gas-rich comets usually is more extended than the dust coma.

From the discussion presented in this paper we conclude that the existence of a class of comets with a polarization at phase angles around $90^{\circ}$ of less than $20 \%$ (Levasseur-Regourd et al. 1996) appears highly doubtful. The degree of polarization in 
so-called gas-rich comets with large dust grains, as evidenced by the absence of superheated grains and lack of a silicate feature in the thermal infrared range, is similar or possibly even higher than in so-called dust-rich comets displaying superheated grains and silicate feature and therefore having dust grains of smaller (submicron) size. A classification of comets according to their polarization requires a more careful consideration of the selected aperture and of the influence of molecular emission transmitted by the filter employed to define the wavelength range of the measurements.

Acknowledgements. We are grateful for generous allocation of observing time at the 2-m telescope of the National Observatory of the Bulgarian Academy of Sciences and at the 2.6-m and 1.25-m telescopes of the Crimean Astrophysical Observatory. N.K. and T.B. have been supported by fellowship grants of the Max Planck Society. K.J. acknowledges a useful discussion with Prof. Per Jensen, University of Wuppertal, Germany, about the vibronic states of the $\mathrm{NH}_{2}$ molecule.

\section{References}

Arpigny, C. 1995, Spectra of Comets: Ultraviolet and Optical Regions, in Laboratory and Astronomical High Resolution Spectra, ed. A. J. Sauval, R. Blomme, \& N. Grevesse, ASP Conf. Ser., 81, 362

Bonev, T., Jockers, K., Petrova, E., et al. 2002, Icarus, 160, 419

Brown, M. E., Bouchez, A. H., Spinrad, H., \& Johns-Krull, C. M. 1996, AJ, 112, 1197

Bunker, P. R., \& Jensen, P. 1998, Molecular Symmetry and Spectroscopy, 2nd Ed. (Ottawa: NRC Research Press)

Chernova, G. P., Kiselev, N. N., \& Jockers, K. 1993, Icarus, 103, 144

Eaton, N., Scarrott, S. M., \& Gledhill, T. M. 1992, MNRAS, 258, 384

Festou, M. C., \& Barale, O. 2000, AJ, 119, 3119

Fink, U., \& Hicks, M. D. 1996, ApJ, 459, 729

Gehrz, R. D., Ney, E. P., Piscitelli, J., et al. 1989, Icarus, 80, 280

Gehrz, R. D., \& Ney, E. P. 1992, Icarus, 100, 162

Geyer, E. H., Jockers, K., Kiselev, N. N., \& Chernova, G. P. 1996, Ap\&SS, 239, 259

Hadamcik, E., \& Levasseur-Regourd, A.-C. 2003, Icarus, 166, 188

Hanner, M. S. 1980, Physical Characteristics of Cometary Dust from Optical Studies, in Solid Particles in the Solar System, ed. I. Halliday, \& B. A. McIntosh (Dordrecht: D. Reidel), 223

Hanner, M. S. 2003, J. Quant. Spectrosc. Radiat. Transf., 79, 695

Hanner, M. S., Knacke, R., Sekanina, Z., \& Tokunaga, A. T. 1985, A\&A, 152, 177

Hanner, M. S., Russell, R. V., Lynch, D. K., \& Brooke, T. Y. 1993 , Icarus, 101, 64

Hanner, M. S., Veeder, G. J., \& Tokunaga, A. T. 1992, AJ, 104, 386

Hanner, M. S., Lynch, D. K., \& Russell, R. V. 1994, ApJ, 425, 274

Harker, D. E., Woodward, C. E., Wooden, D. H., et al. 1999, AJ, 118, 1423

Heiles, C. 2000, AJ, 119, 923

Hsu, J.-C., \& Breger, M. 1982, ApJ, 262, 732

Jensen, P., Kraemer, W. P., \& Bunker, P. R. 2003, Mol. Phys., 101(4-5), 613

Jewitt, D. 2004, AJ, 128, 3061

Jockers, K. 1997a, Exp. Astron., 7, 305

Jockers, K. 1997b, Earth Moon and Planets, 79(1-2), 221

Jockers, K., Credner, T., Bonev, T., et al. 2000, Kinematics and Physics of Celestial Bodies Suppl., 3, 13

Kelley, M. S., Woodward, C. E., Jones, T. E., et al. 2004, AJ, 127, 2398

Kikuchi, S. 1983, Proc. 16th Lunar and Planetary Symp., 16, 36
Kikuchi, S., Okazaki, A., Kondo, M., \& Hirata, A. 1990, Proc. of 23th ISAS Lunar and Planetary Symp., 39

Kimura, H. 2001, J. Quant. Spectrosc. Radiat. Transf., 70, 581

Kiselev, N. N. 2003, Doctoral Thesis, Kharkov University, Ukraine

Kiselev, N. N., Jockers, K., Rosenbush, V. K., et al. 2000, Planet. Space Sci., 48, 1005

Kiselev, N. N., Jockers, K., Rosenbush, V. K., \& Korsun, P. P. 2001, Astron. Vestn., 35(6), 531 (Sol. Syst. Res. (engl. Transl.), 35(6), 480)

Kiselev, N. N., Jockers, K., \& Rosenbush, V. K. 2002, Earth Moon and Planets, 90, 167

Kiselev, N. N., Jockers, K., \& Bonev, T. 2004, Icarus, 168, 385

Krishna Swamy, K. S. 1986, Physics of Comets (Singapore: World Scientific)

Le Borgne, J. F., Leroy, J. L., \& Arnaud, J. 1986, Polarimetry of Molecular Bands in Comet P/Halley and Hartley-Good, in Proc. of 20th ESLAB Symp. Exploration of Halley's Comet, ed. E. J. Rolfe, \& B. Battrick, ESA SP-250, 571

Le Borgne, J. F., \& Crovisier, J. 1987, Polarization of Molecular Fluorescence Bands in Comets: Recent Observations and Interpretation, in Diversity and Similarity of Comets, ed. E. J. Rolfe, \& B. Battrick, ESA SP-278, 171

Levasseur-Regourd, A. C., Hadamchik, E., \& Renard, J. B. 1996, A\&A, 313, 327

Lynch, D. K., Hanner, M. S., \& Russell, R. W. 1992, Icarus, 97, 269

Mann, I., Kimura, H., \& Kolokolova, L. 2004, J. Quant. Spectrosc. Radiat. Transf., 89, 291

Myers, R. V., \& Nordsieck, K. H. 1984, Icarus, 58, 431

Ney, E. P. 1982, Optical and Infrared Observations of Bright Comets in the Range $0.5 \mu \mathrm{m}$ to $20 \mu \mathrm{m}$, in Comets, ed. L. L. Wilkening (Tucson: Univ. Arizona Press), 323

Osip, D. J., Schleicher, D. G., \& Millis, R. L. 1992, Icarus, 98, 115

Petrova, E. V., Jockers, K., \& Kiselev, N. N. 2000, Icarus, 148, 526

Petrova, E. V., Tishkovets, V. P., \& Jockers, K. 2004, Astron. Vestn., 38(4), 354 (Sol. Syst. Res. (engl. transl.), 38(4), 309)

Piirola, V. 1973, A\&A, 27, 383

Piirola, V. 1988, Simultaneous Five-Colour (UBVRI) Photopolarimeter, in Polarized Radiation of Circumstellar Origin, ed. G. V. Coyne, S. J. A. M. Magalhaes, A. F. J. Moffat, et al. (Vatican City State/Tucson, Vatican Observatory/University of Arizona Press), 735

Reach, W. T., Sykes, M. V., Lien, D., \& Davies, J. K. 2000, Icarus, 148,80

Rosenbush, V. K., Rosenbush, A. E., \& Dementev, M. S. 1994, Icarus, 108, 81

Rosenbush, V. K., Tarashchuk, V. P., Kiselev, N. N., et al. 1997, Astron. Vestn., 31(6), 448

Sekanina, Z. 1988, AJ, 95, 911

Serkowsky, K. 1974, Polarimeters for Optical Astronomy, in Planets, Stars and Nebulae Studied with Photopolarimetry, ed. T. Gehrels (Tucson: Univ. Arizona Press), 135

Shakhovskoy, N. M., Andronov, I. L., \& Kolesnikov, S. V., et al. 2001, Izv. Krymskoi Astrofiz. Obs., 97, 91

Shakhovskoy, N. N., \& Efimov, Yu. S. 1972, Izv. Krymskoi Astrofiz. Obs., 45, 90

Tishkovets, V. P., Petrova, E. P., \& Jockers, K. 2004, J. Quant. Spectrosc. Radiat. Transf., 86, 241

Truhins, K., Al Wahabi, Z. T., Auzinsh, M., et al. 1997, J. Chem. Phys., 106, 3477

Wardle, J. F. C., \& Kronberg, P. P. 1974, ApJ, 194, 249

West, R. A. 1991, Appl. Opt., 30, 316

Xing, Z., \& Hanner, M. 1997, A\&A, 423, 805 